\title{
MODELO BIM/FM PARA EDIFÍCIO UNIVERSITÁRIO CONCEBIDO A PARTIR DE PROJETO EXISTENTE
}

\author{
SILVA, Marcus Vinicius Rosário da \\ Universidade Federal Fluminense, e-mail: marcusrosario@yahoo.com.br \\ MEIRIÑO, Marcelo Jasmim \\ Universidade Federal Fluminense, e-mail: marcelojm@id.uff.br \\ MENEGOTTO, José Luis \\ Universidade Federal do Rio de Janeiro, e-mail: jlmenegotto@poli.ufrj.br
}

\begin{abstract}
RESUMO
Os modelos BIM têm potencial de melhorar, de forma eficaz, as limitações dos desenhos 2D. Para aplicações em Facility Management (FM), o uso da nova tecnologia tem como um dos desafios superar lacunas relacionadas à qualidade da informação entregue nas etapas precedentes ao uso e manutenção. O objetivo principal deste trabalho foi desenvolver um modelo BIM/FM para um edifício educacional de ensino superior. Foi criado, inicialmente, como produto de uma disciplina do mestrado - já concluída. Utilizou-se como métodos científicos a pesquisa documental, estudo de caso e revisão narrativa. Nos resultados foram apresentados: (a) construção de informações geométricas; e (b) investigação sobre interoperabilidade e integração de dados. A pesquisa contribui para a aplicabilidade do BIM, com respectiva gestão do conhecimento relacionado aos edifícios existentes em campus universitários brasileiros.
\end{abstract}

Palavras-chave: Gestão do Conhecimento, Tecnologia da Informação e Comunicação, Ciclo de Vida, Ambiente Construído, Uso e Manutenção.

\begin{abstract}
$B I M$ models have the potential to improve, effectively, the limitations of $2 D$ drawings. For its application in Facility Management (FM) the use of the new technology has as challenge overcome gaps related to the quality of the information delivered in the previous stages of use and maintenance. The main objective of this paper was to develop a BIM model for an educational building of higher education. The model was initially created as a product of a subject in the master's course already completed. Documentary research, case study, and narrative review were used as scientific methods. In the results were presented: (a) geometric information; and (c) interoperability and data integration. This study contributes to the applicability of BIM and respective knowledge management and the related to existing buildings on Brazilians universities campus.
\end{abstract}

Keywords: Knowledge Management, Technology Information Communication, Life cycle, Built Environment, Use and Maintenance.

\section{INTRODUÇÃO}

Os campi universitários brasileiros, alinham-se ao conceito de megaestrutura definida por Banham (2001), constituídas por edifícios lineares e/ou em malha. A proposta brutalista, exemplificada pelo estudo de caso do presente trabalho, é um dos elementos que associam o uso de concreto aparente (campo estético) e exposição dos recursos estruturais (campo tecnológico).

SILVA, M. V. R.; MEIRIÑO, M. J.; MENEGOTTO, J. L. Modelo BIM/FM para edifício universitário concebido a partir de projeto existente. In: SIMPÓSIO BRASILEIRO DE QUALIDADE DO PROJETO NO AMBIENTE CONSTRUÍDO, 6., 2019, Uberlândia. Anais... Uberlândia: PPGAU/FAUeD/UFU, 2019. p 703-713. DOI https://doi.org/10.14393/sbqp19065. 
Atualmente, os edifícios universitários padecem com a ação do tempo e da falta de manutenção (ALBERTO, 2013; MAHLER, 2015).

Com a tecnologia de Modelagem da Informação da Construção (do inglês, Building Information Modelling - BIM), muitas limitações podem ser melhoradas de forma eficaz. Há estudos focados na aplicação da nova tecnologia durante a fase de uso, operação e manutenção, mas poucas pesquisas consideraram o desenvolvimento do plano de execução BIM. Tendo, como uma das principais barreiras, a falta de qualidade da informação entregue (LIN; CHEN; HUANG; HONG, 2016; ZADEH; WANG; CAVKA; STAUB-FRENCH; POTTINGER, 2016). Neste contexto, o objetivo do trabalho é desenvolver um modelo BIM/FM para um edifício educacional, localizado no campus universitário.

Atualmente, a função organizacional de FM é conceituada como a integração de "pessoas, lugares e processos dentro do ambiente construído com o propósito de melhorar a qualidade de vida das pessoas e a produtividade do negócio central" (ISO, 2017). Segundo a norma alemã DIN 32736, a base de sustentação do FM é a gestão dos espaços, sendo os seus aspectos principais divididos em: (a) gestão técnica - atividades de operação, manutenção e documentação dos sistemas prediais; (b) gestão de infraestrutura - atividades de conservação; e (c) gestão comercial responsável pela administração (DIN, 2000).

As organizações envolvidas em FM têm a oportunidade de usar o BIM como repositório de conhecimento para documentar as informações das instalações em evolução e para apoiar à tomada de decisão pelo Facility Manager durante a vida útil de uma instalação. Mesmo que o BIM seja capaz de melhorar a qualidade de vida no ambiente de trabalho, a resistência à mudança é resultado de percepções diferentes de ideias, motivos, planos ou prioridades (AZIZ; NAWAWI; ARIFF, 2016).

Dentro de cada departamento acadêmico, há pessoal especificamente responsável por tarefas relacionadas ao gerenciamento de espaço. Frequentemente, essas pessoas solicitam informações dos objetos inventariados da unidade ou informes relativos a espaços e recursos humanos dos departamentos (ZADEH; CAVKA; SATUB-FRENCH, 2016).

Facility Managers consideram que a aplicação do BIM reduz o tempo de busca destas informações e as suas correspondentes visualizações. A transmissão de informações relacionadas ao MEP (do inglês, Mechanical, electrical and plumbing) criadas na fase de projeto e construção para a fase de Operação e Manutenção ( $O$ \& $M$ ) melhora a eficiência da gestão, alterando os procedimentos convencionais para aplicar a tecnologia $(\mathrm{HU}$; TIAN; LI; ZHANG, 2018).

Modelos BIM para edifício(s) tem potencial para auxiliar na gestão da transferência de informação entre operações em tempo real e sistemas de monitoramento e o tratamento da incerteza com base em documentação de construção incompleta (MCARTHUR, 2015). Para que o processo possa ser bem-sucedido é necessário que sejam desenvolvidos ordenamentos cada vez mais apurados, com os quais se possa melhorar o correto cruzamento das informações. Recentemente Farghaly (2018) propus uma taxonomia de 40 parâmetros estruturados em cinco categorias principais: 1) espaço/localização; 2) despesas de capital (CAPEX); 3) especificações; 4) 
garantias; e, 5) despesas operacionais (OPEX) do ativo. De acordo com os autores do estudo, embora possa conter lacunas, ela condensa esforços de grupos internacionais de especialistas e visa à implementação efetiva do BIM na prática de gestão de ativos (FARGHALY et al., 2018).

\section{METODOLOGIA}

\subsection{Pesquisa documental}

Inicialmente, a pesquisa documental foi conduzida a fim de esgotar todas as pistas capazes de fornecer informações relevantes sobre a edificação - objeto de estudo (SÁ-SILVA; ALMEIDA; GUINDANI, 2009).

\subsection{Estudo de Caso}

Posteriormente, o estudo de caso foi aplicado como método de pesquisa qualitativa para coletar dados a partir dos eventos reais, com o intuito de explicar, explorar e descrever os fenômenos atuais inseridos no próprio contexto (YIN, 2009).

\subsection{Pesquisa bibliográfica}

Por fim, foi realizado análise da literatura já publicada em forma de livros, artigos e literatura cinzenta (teses, dissertações e trabalhos apresentados em congressos) utilizando o método de revisão narrativa (CORDEIRO et al., 2007).

\section{RESULTADOS E DISCUSSÃO}

\subsection{Construção de Informação Geométrica}

Como resultado da pesquisa documental, realizada no acervo da Prefeitura Universitária (PU), os autores obtiveram acesso a planta de situação e as plantas baixas em arquivo CAD (Figura 1).

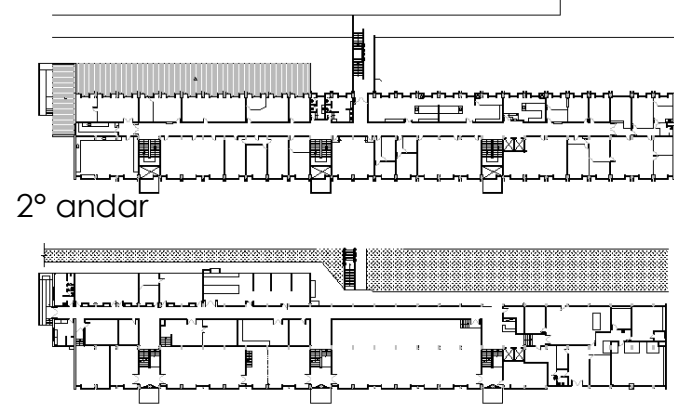

Térreo

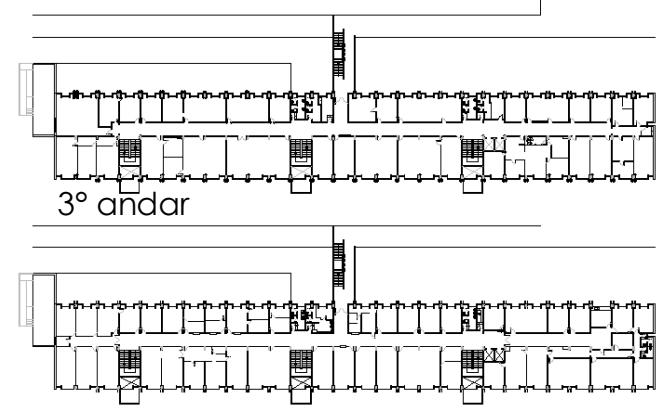

$4^{\circ}$ andar

Figura 1 - Plantas baixas da edificação -

Fonte: Adaptado da PU (2018)

Não havia documentos arquivados, como cortes, elevações, detalhes e projetos complementares e/ou suplementares, tais quais projeto estrutural, hidrossanitário, elétrico, telefonia e combate a incêndio.

Para o estudo de caso, foram coletadas informações da edificação, tais quais obras/reformas executadas ao longo dos anos, mudanças de uso dos ambientes decorrentes da liberação para cursos de pós-graduação e 
laboratórios de pesquisa, além de quantitativo de ativos por ambiente e especificação dos revestimentos.

O modelo 3D foi concebido no software Autodesk Revit a partir das informações supracitadas, conforme Figura 2.

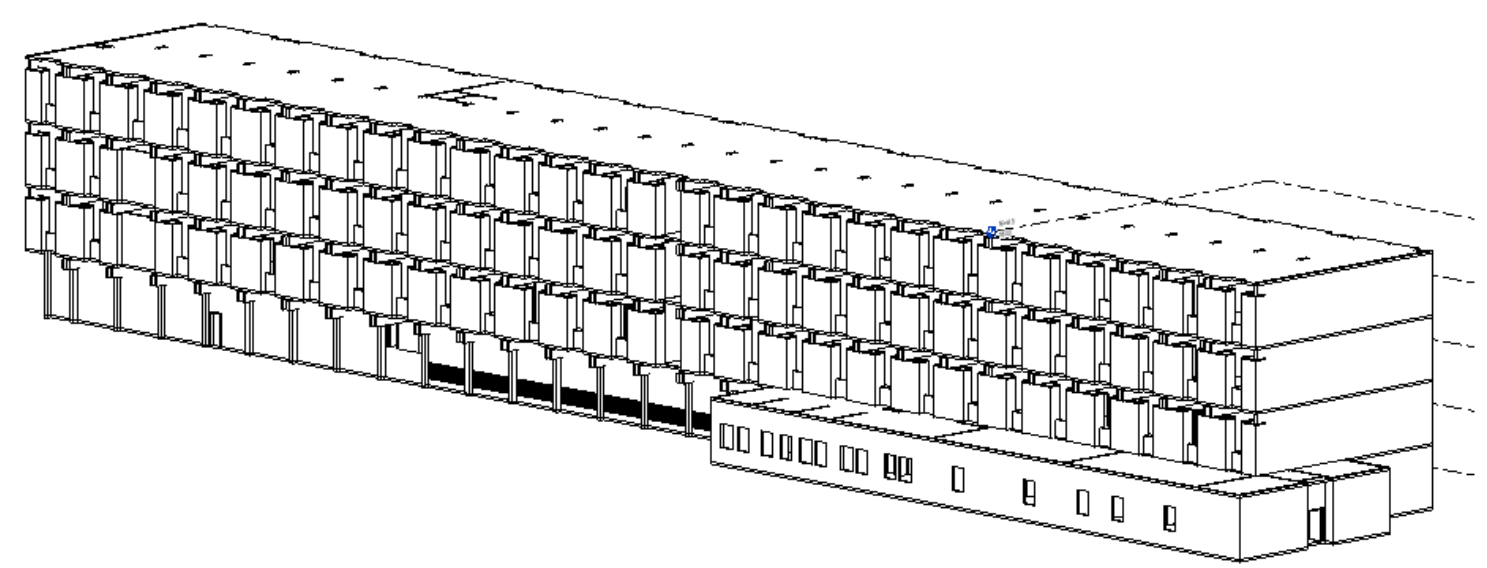

Figura 2 - Modelo BIM do edifício universitário -

Fonte: Autores (2019)

O modelo em escala de vista 1:100, com baixo nível de detalhe para a disciplina de Arquitetura. Tendo seus níveis modelados com restrição superior nível acima imediatamente adjacente. Deslocamentos nas vedações verticais foram realizados devido existência de vigas. A modelagem de informação da edificação foi concebida em caráter preliminar e passará por novas fases para refinamento da modelagem e inserção de componentes adicionais.

O modelo-base permitiu o lançamento da especificação dos revestimentos, ativos e alterações realizadas nos ambientes durante o decorrer dos anos por recorte temporal, proporcionando uma melhoria na gestão do conhecimento relacionada ao edifício do campus universitário.

Há informações do período de vida útil que foram perdidas devido falta de documentação e transmissão oral. Uma força tarefa adicional deverá ser feita para realização de um as-built dos sistemas prediais, e lançamento no modelo. Sido identificado a oportunidade de parceria com as empresas júniores dos cursos de engenharia para sua execução.

\subsection{Investigação de Interoperabilidade e integração de Dados}

Os arquivos IFC contém a informação do edifício, entretanto não nos interessa manusear toda essa informação, mas sim parte dela. Desta forma, o COBie entra como um subconjunto das informações necessárias para a gestão e operação do edifício. O COBie é um Model View Definition (MVD), homologado pela BuildingSMART (MAZIONE, 2014).

O Construction Operations Building Information Exchange (COBIE), um formato de dados de planilha para manipular a informação do edifício, foi considerado como uma das soluções que facilitariam o processo de transferência de informações de sistemas BIM para Computer Aided Facilities Management (CAFM). Várias aplicações de CAFM oferecem funções para 
exportar / importar informações para o formato COBie (EAST, 2007; LEE et al., 2013).

Para viabilizar a interoperabilidade, $O$ Industry Fundation Classes (IFC) surgiu como um modelo de representação de dados público e não proprietário, desenvolvido para a AEC/FM (Arquitetura, Engenharia, Construção e Facility Management (CAPESTRINI et al., 2015; SPÍNOLA; MEDEIROS; MENEZES, 2015). Uma linha do tempo foi criada para sintetizar o desenvolvimento do IFC ao longo dos anos (Figura 3).

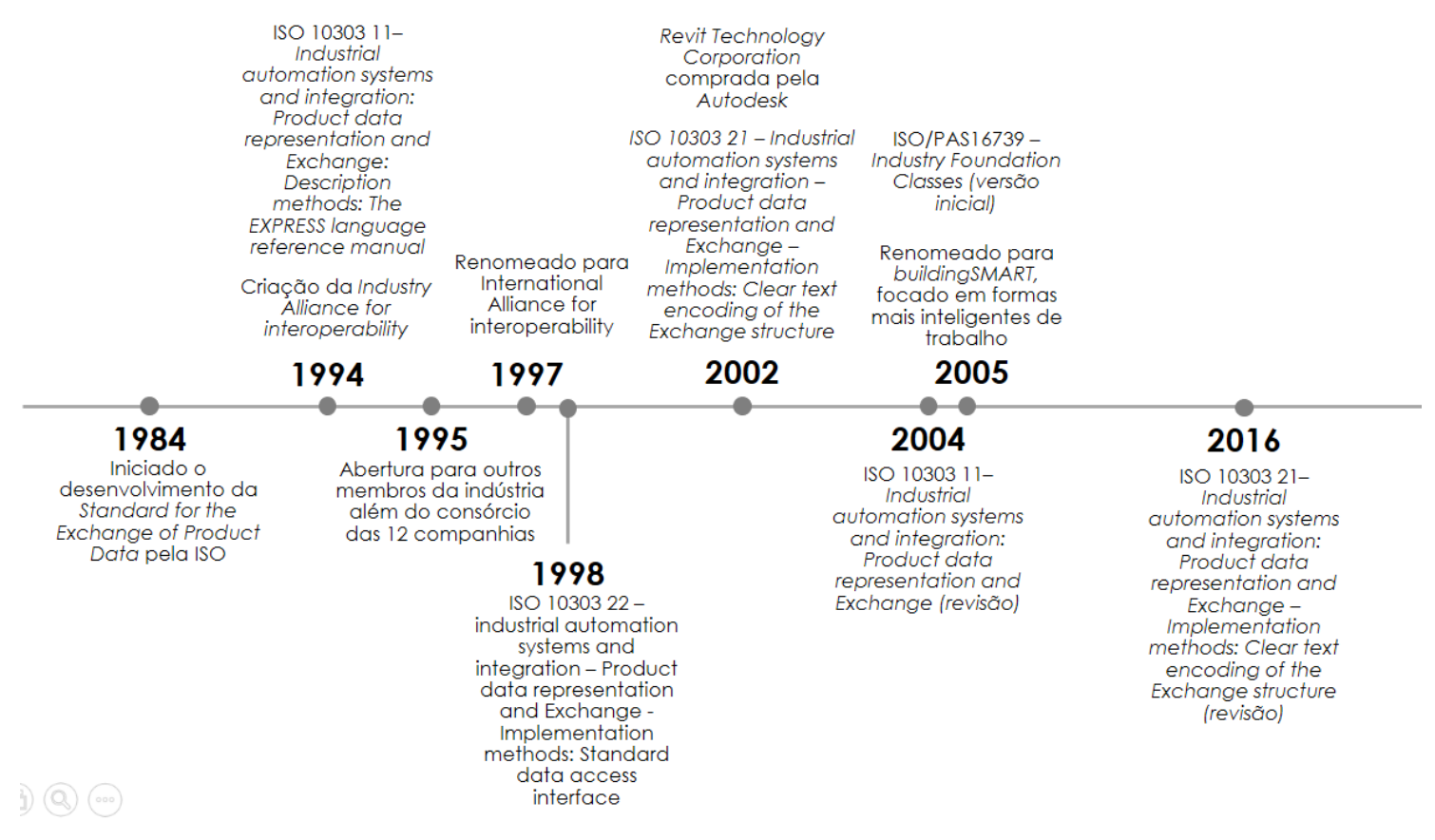

Figura 3 - Linha do tempo -

Fonte: Autores a partir de compilação de dados em Manzione (2014)

O COBie é um Model View Definition (MVD), homologado pela BuildingSMART e define um subconjunto do Esquema da IFC necessário para satisfazer a troca de requisitos definidos no Information Delivery Manual (IDM). Assim, no Quadros 1 são apresentados os requisitos IFC pertinentes ao COBie, e no Quadro 2 seus respectivos objetivos (MAZIONE, 2014; PINHEIRO et al., 2016).

Durante a última década, vem sendo realizados diversos esforços para integrar sistemas BIM dentro do conjunto de tecnologias da Web semântica (WS). Assim como o BIM, a WS é uma tecnologia que se insere no domínio dos ambientes de modelagem de dados e de conhecimento. Através de seus diversos instrumentos (URIs, RDFs, ontologias, etc.) a WS permite vincular e interoperar com dados distribuídos e heterogêneos gerados em diversos domínios de conhecimento, além de empregar mecanismos de raciocínio lógico para inferir novos conhecimentos de modo automático e autônomo (BERNERS-LEE et al., 2001; ISO, 2004; HITZLER et al., 2009).

Essas características motivaram o trabalho de ampliação dos esquemas IFC em uma ontologia OWL (Ontology Web Language) específica, o ifcOWL. Ao criar uma ifc $O W L$ como referência é proposto um aprimoramento do padrão de interoperabilidade BIM para integrar sua aplicabilidade a sistemas de processamento inteligente (PAUWELS, 2015; ISO, 2016). 
Quadro 1 - Requisitos IFC

\begin{tabular}{|c|c|c|c|c|c|c|c|c|}
\hline \multirow[b]{2}{*}{ Grupo } & \multirow[b]{2}{*}{ Requisitos } & \multicolumn{7}{|c|}{ Especificação } \\
\hline & & 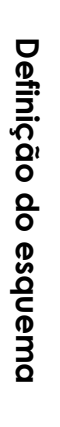 & 궁 & $\begin{array}{l}\frac{m}{3} \\
\frac{0}{0} \\
\frac{2}{0} \\
\frac{0}{D}\end{array}$ & 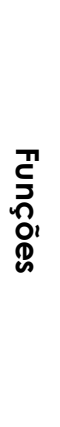 & $\begin{array}{l}\text { D্ } \\
\stackrel{0}{1} \\
\text { 은 }\end{array}$ & 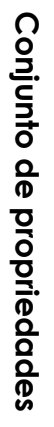 &  \\
\hline \multirow{4}{*}{$\begin{array}{c}\text { Esquema de dados } \\
\text { gerais }\end{array}$} & IFC kernel & $x$ & $x$ & $x$ & $x$ & $x$ & $x$ & \\
\hline & IFC Control Extention & $x$ & $x$ & $x$ & & & & \\
\hline & IFC Process Extention & $x$ & $x$ & $x$ & & & $x$ & \\
\hline & IFC Product Extention & $x$ & $x$ & $x$ & $x$ & & $x$ & \\
\hline \multirow{2}{*}{$\begin{array}{c}\text { Esquemas de dados } \\
\text { de elemento } \\
\text { compartilhado }\end{array}$} & IFC Shared Facilities Elements & $x$ & $x$ & $x$ & & & $x$ & \\
\hline & IFC Shared MGMT Elements & $x$ & $x$ & $x$ & & & $x$ & \\
\hline $\begin{array}{l}\text { Esquema de dados } \\
\text { específicos de } \\
\text { domínio }\end{array}$ & IFC Construction MGMT Domain & $x$ & $x$ & $x$ & & & $x$ & $x$ \\
\hline \multirow{8}{*}{$\begin{array}{c}\text { Esquemas de dados } \\
\text { de definição de } \\
\text { recursos }\end{array}$} & IFC Actor Resource & $x$ & $x$ & $x$ & & & & \\
\hline & IFC Constrain Resource & $x$ & $x$ & $x$ & & & & \\
\hline & IFC Date Time Resource & $x$ & $x$ & $x$ & & & & \\
\hline & IFC External Reference & $x$ & $x$ & $x$ & & & & \\
\hline & IFC Measure Resource & $x$ & $x$ & $x$ & $x$ & & & \\
\hline & IFC Property Resource & $x$ & $x$ & $x$ & $x$ & & & \\
\hline & IFC Quantity Resource & $x$ & & $x$ & $x$ & & & \\
\hline & IFC Utility Resource & $x$ & $x$ & $x$ & & & & \\
\hline
\end{tabular}

Fonte: Autores

Niknam e Karshenas (2015) propuseram uma ontologia compartilhada que contém apenas os principais componentes de um modelo de construção (paredes, espaços, elementos e pisos). Dependendo do caso de uso, essa ontologia compartilhada tem dados expandidos seguindo uma ontologia de projeto (NIKNAM; KARSHENAS, 2015; PAUWELS; ROXIN, 2016).

Para modelagem de uma ontologia aplicada à FM, deve-se: (a) garantir a consistência do conhecimento desde a fase de construção até a fase de gestão técnica (pós-entrega); e (b) fornecer, para cada agente envolvido no projeto, uma "visão" atualizada do edifício (PITTET; CRUZ; NICOLLE, 2014).

Os atores de FM utilizam softwares heterogêneos, sendo uma oportunidade para uso de subontologias especializadas de acordo com as especificidades dos processamentos envolvidos; reduzindo o tempo dedicado à consulta dos bancos de dados (PITTET; CRUZ; NICOLLE, 2014). 
Quadro 2 - objetivos dos requisitos IFC correlacionados ao COBie

\begin{tabular}{|c|c|}
\hline Requisitos IFC & Objetivo \\
\hline kernel & $\begin{array}{l}\text { Capturar construções gerais, que são basicamente fundadas por } \\
\text { seus diferentes significados semânticos na compreensão comum de } \\
\text { um modelo de objeto (objeto, propriedade e relacionamento) }\end{array}$ \\
\hline Process Extention & $\begin{array}{c}\text { Capturar informações que suportem o mapeamento de processos e } \\
\text { planejamento e agendamento de trabalho e procedimentos e } \\
\text { recursos necessários para executar o trabalho }\end{array}$ \\
\hline Product Extention & $\begin{array}{c}\text { Capturar anotações adicionais a um modelo ou plano de } \\
\text { construção (texto explicativo, dimensionamento, etc.) e uma porta, } \\
\text { fornecendo conectividade entre elementos por meio de uma grade } \\
\text { com restrição para os elementos }\end{array}$ \\
\hline $\begin{array}{l}\text { Shared Facilities } \\
\text { Elements }\end{array}$ & $\begin{array}{l}\text { Agrupar elementos de mobiliário de sistema em itens de mobiliário } \\
\text { individuais, identificação de ativos, e inventário de objetos (ativos, } \\
\text { móveis, e objetos espaciais dentro de inventários separados) }\end{array}$ \\
\hline $\begin{array}{l}\text { Shared MGMT } \\
\text { Elements }\end{array}$ & $\begin{array}{l}\text { Capturar informações que suportam o controle do escopo, custo e } \\
\text { tempo do projeto. Fornece suporte para troca e compartilhamento } \\
\text { de informações mínimas sobre os assuntos em escopo }\end{array}$ \\
\hline $\begin{array}{c}\text { Construction MGMT } \\
\text { Domain }\end{array}$ & $\begin{array}{c}\text { Capturar informações que suportem processos de negócios e } \\
\text { requisitos de recursos específicos que estejam totalmente dentro do } \\
\text { domínio de interesse do Gerente do edifício }\end{array}$ \\
\hline
\end{tabular}

Fonte: Elaborado pelos autores a partir de buildingSMART (2019)

Farias, Roxin e Nicolle (2015) propõem um método para conceber de forma semiautomática uma ontologia OWL para padrão COBie, reduzindo as barreiras para adoção do BIM a partir da falta de flexibilidade, especialização técnica e custo de software (Figura 4).

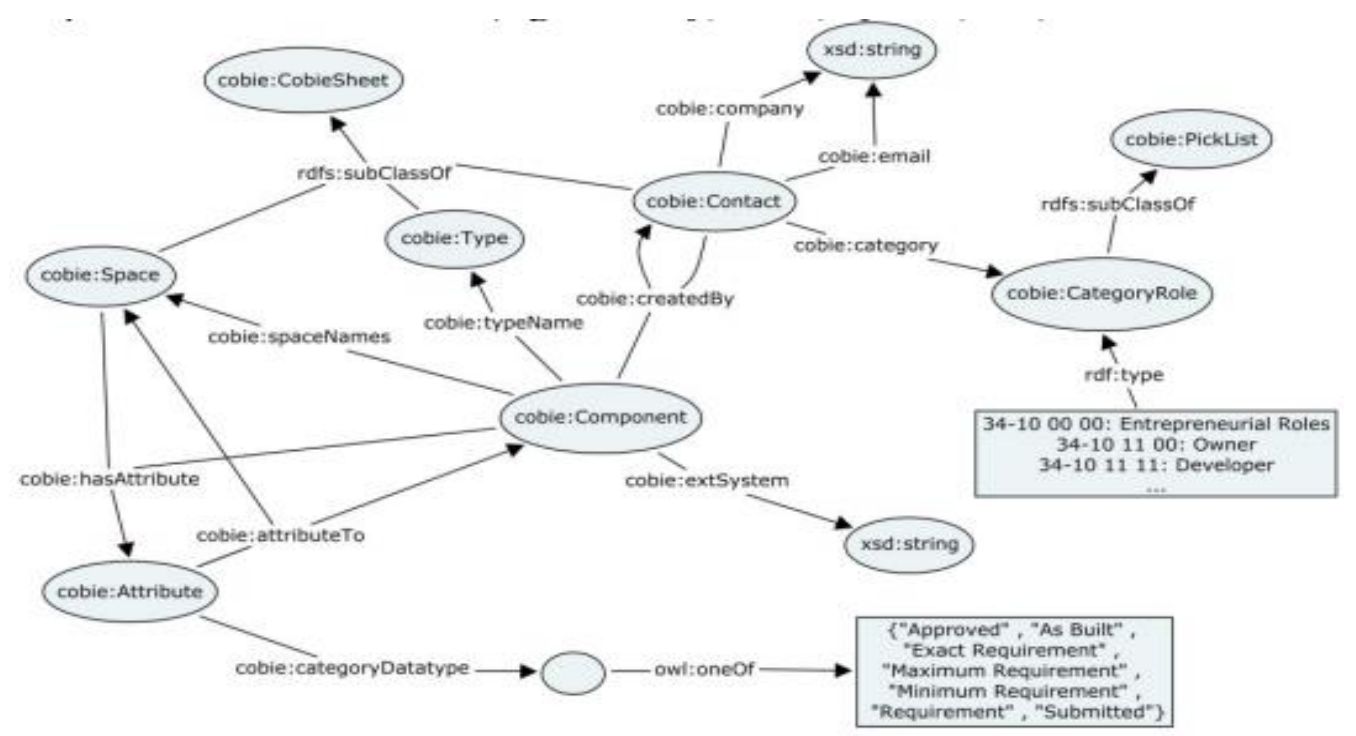

Figura 4 - Ontologia de COBieOWL -

Fonte: FARIAS; ROXIN; NICOLLE (2015)

Resumidamente, a Figura 5 é a estrutura metodológica geral da informação bidirecional entre BIM e FM baseada em ontologia. Nesta estrutura proposta por Chen et al. (2018), o esquema da IFC é explorado em primeiro lugar para encontrar as entidades que representam componentes e espaços de 
construção. Existem alguns documentos necessários gerados no período de O\&M, como ordens de serviço de manutenção e especificações, que não são armazenados em modelos BIM e, portanto, não podem ser fornecidos a partir de modelos BIM. As informações sobre documentos, recursos e tarefas são representadas no esquema de dados COBie, mas não são representadas no esquema IFC (CHEN et al., 2018).

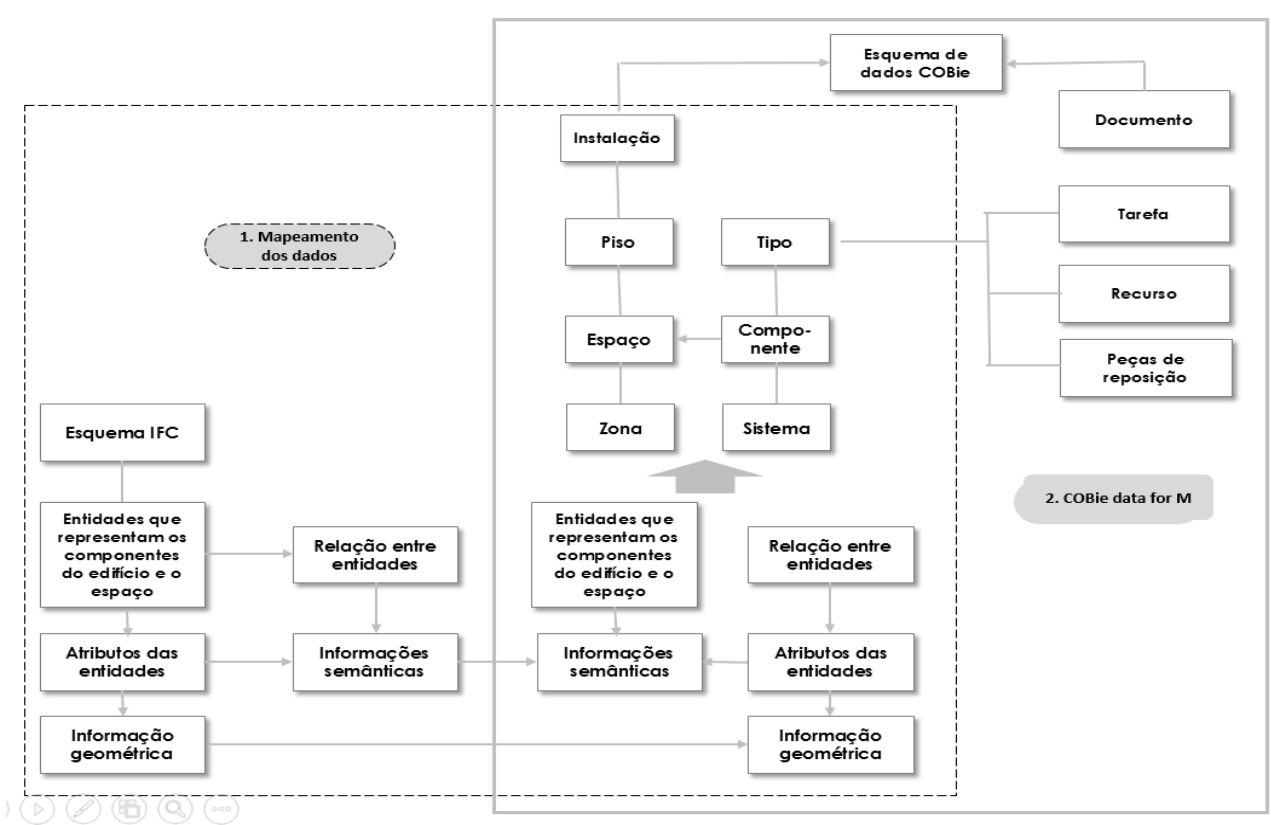

Figura 5 - Integração de dados entre BIM/FM baseado em ontologia Fonte: CHEN; CHEN; CHENG (2018, tradução dos autores)

\section{CONSIDERAÇÕES FINAIS}

O trabalho resultou na construção de informação geométrica de um edifício dedicado ao ensino superior e de estética brutalista, contido em um campus universitário, alinhada ao conceito de megaestrutura; e investigação sobre interoperabilidade e integração de dados por meio de ontologia.

A modelagem 3D foi constituída com base no acervo de plantas existentes do local e complementação das informações por meio de observação da situação da edificação, no que tange seu estado de uso, conservação e manutenção - diretamente relacionadas às atividades de Facility Management. Oportunidades de melhoria do modelo foram identificadas para suprir lacunas da qualidade de informações entregues, tais quais realização de as-built dos sistemas prediais.

Investigar ambos os aspectos, tanto o da interoperabilidade como o da vinculação de dados, são de suma importância para a fase de Operação e Manutenção. A função organizacional de FM é o elemento principal nesta fase do ciclo de vida. O FM é composto por equipes interdisciplinares de profissionais que atuam na gestão técnica, de infraestrutura, comercial e espacial. Neste cenário, torna-se relevante considerar que cada setor utiliza softwares heterogêneos, sendo necessário o esforço para gerar interoperabilidade e vinculação de dados. 
Assim, procurou-se identificar iniciativas de desenvolvimento de ontologias que suprissem as lacunas e compreendesse as limitações de cada um dos diferentes profissionais de FM. Atuar com ifcOWL e COBie foi identificado como um aspecto relevante neste cenário. Outro ponto, é a observância do nível de acesso a informação, constituindo por subontologias, que tornarão o acesso a informação pretendida mais ágil.

Com relação à organização dos elementos funcionais do edifício modelado é necessário que a organização das partes, assim como as nomenclaturas de tipos e propriedades paramétricas sejam pensadas em paralelo a uma definição formal e ontológica do edifício, a exemplo dos estudos conduzidos por Rasmunssen et al. (2018) nos quais se procura propor uma ontologia geral topologicamente pensada para a construção (Building Topology Ontology, BOT).

\section{REFERÊNCIAS}

ALBERTO, K.; C. Interfaces Brutalistas: Megaestruturas universtárias. Anais do X Seminário DOCOMOMO Brasil. Arquitetura Moderna e Internacional: conexões brutalistas 1955-75. Curitiba, 15-18 out, PUCPR, 2013.

AZIZ, N. D.; NAWAWI, A. H.; ARIFF, N. R. M. Building Information Modelling (BIM) in Facilities Management: Opportunities to be considered by Facility Managers.

AMER International Conference on Quality of Life, AicQoL 2016. Indonesia, 2016.

BANHAM, R. Megaestruturas: Futuro Urbano del passado reciente. Barcelona: Editora Gustavo Gilli, 2001.

BERNERS-LEE, T.; HENDLER, J.; LASSILA, O. The semantic web. Scientific American, 2001.

BUILDINGSMART. Building without barreirs: The business case for interoperability. Disponível em: <https://www.buildingsmart.org/wpcontent/uploads/2019/04/buildingSMART-A5_Brochure_V10_.pdf>. Acesso em: 02 junho de 2019.

CAPESTRINI, T. F.; GARRIDO, M. C.; MENDES JR, R.; SCHEER, S.; GREINTAS, M. C. D. Entendendo BIM. 1. Ed. Curitiba: UFPR, 2015.

CHEN, W.; CHEN, K.; CHENG, J. C. P. Towards an Ontology-based Approach for Information Interoperability Between BIM and Facility Management. Advanced

Computing Strategies for Engineering: 25 th EG-ICE International Workshop 2018. Lausanne, 2018.

CORDEIRO, A. M.; OLIVEIRA, G. M.; RENTERIA, J. M.; GUIMARÃES, C. A. Revisão Sistemática: uma revisão narrativa. Comunicação Científica. 2007

DEUTSCHES INSTITUT FÜR NORMUNG (DIN). DIN 32736: GebäUdemanagement. Beriln, 2000.

FARGHALY, K.; ABANDA, H.; VIDALAKIS, C.; WOOD, G. BIM for Asset Management: A taxonomy of non-geometric BIM data for asset management. Conference EG-ICE 24 ${ }^{\text {th }}$ Workshop, Reino Unido, jul., 2017.

HITZLER, P. et al. OWL 2 Web Ontology Language (Primer). W3C, 11 Dec. 2012. 
HU, Z. Z.; TIAN, P. L.; LI, S. W.; ZHANG, J. P. BIM-based integrated delivery technologies for intelligent MEP management in the operation and maintenance phase. Advances in Engineering Software, v. 115, 2018. INTERNATIONAL STANDARD ORGANIZATION (ISO). ISO 41.011: Facilities Management - Vocabulary. UK, 2017.

. ISO 10303-11: Industrial automation systems and integration - Product data representation and exchange - Part 11: Description methods: The EXPREE language reference manual. 2004.

ISO 10303-21: Industrial automation systems and integration - Product data representation and exchange - Part 21: Implementation methods: Clear text encoding of the exchange structure, 2016.

LIN, Y. C.; CHEN, Y. P.; HUANG, W. T.; HONG, C. C. Development of BIM execution plan for BIM Model Management during the pre-operation phase: a case study. Buildings, v. 6, n. 8, 2016.

MAHLER, C. R. Territórios universitários: tempos, espaços, formas. Tese (Doutorado) - Faculdade de Arquitetura e Urbanismo, Universidade de Brasília, Brasília, 2015.

MANZIONE, L. Proposição de uma estrutura conceitual de gestão do processo de projeto colaborativo com o uso do BIM. Tese (Doutorado) - Escola Politécnica, Universidade de São Paulo, São Paulo, 2013.

MCARTHUR, J. J. A building information management (BIM) framework and supporting case study for existing building operations, maintenance and sustainability. International Conference on Sustainable Design, Engineering and Construction. 2015.

NIKNAM, M.; KARSHENAS, S. Integrating distributed sources of information for construction cot estimating using semantic web and semantic web servisse Technologies. Automation in construction, v. 57, 2015.

PAUWELS, P.; ROXIN, A. SimpleBIM: from full ifcOWL graphs to simplified building graphs. Proceedings of the $11^{\text {th }}$ European Conference on Product and Process Modelling, 2016.

PAUWELS, P.; ROXIN, A.; NICOLLE, C. ifcWOD, semantically adapting IFC model relations into OWL properties. Proceedings of the $32^{\text {nd }}$ International CIB W78 Conferece, Eindhouven, 2015.

PINHEIRO, S.; O'DONNELL, J.; WIMMER, R.; BAZJANAC, V.; MUHIC, S.; MAILE, T.; FRISCH, J.; TREEEK, C. Model view definition for advanced building energy performance simulation.

PITTET, P.; CRUZ, C.; NICOLLE, C. A strectural mathcal ( SHOIN(D) Ontology model for ge modelling. Lecture notes in computer Science, v. 8186, 2013.

RASMUNSSEN, M. H.; BONDUEL, M.; HVIID, C.; KARLSøJ, J. Managing Space Requirements of New Buildings Using Linked Building Data Technologies. 12th European Conference on Product and Process Modelling. Copenhagen, Denmark, 2018.

SÁ-SILVA, J. R.; ALMEIDA, C. D.; GUINDANI, J. F. Pesquisa documental: pistas teóricas e metodológicas. Revista Brasileira de História \& Ciências Sociais, v. I, n. 1, p. 1-15, Rio Grande do Sul, jul. 2009. 
SPÍNOLA, G. B.; MEDEIROS, N. N.; MENEZES, G. L. B. Arquivos IFC, uma ideia de futuro. X Congresso Norte Nordeste de Pesquisa e Inovação. 2015 YIN, R. K. Case study research, design and methods (applied social research methods). Sage Publications, 2009.

PU. PREFEITURA UNIVERSITÁRIA. Bloco E completo. Out. 2018. Arquivo dwg. $1,08 \mathrm{MB}$.

ZADEH, P. A.; CAVKA, H. B.; STAUB-FRENCH, S. BIM information quality analysis for Space Management. $16^{\text {th }}$ International Conference on Computing in Civil and Building Engineering, Japão, 2016.

ZADEH, P. A.; WANG, G.; CAVKA, H. B.; STAUB-FRENCH S.; POTTINGER, R. Information Quality Assessment for Facility Management. Advanced Engineering informatics, v. 33, p. 181-205, 2017. 